\title{
EDITORIAL
}

For reprint orders, please contact: reprints@futuremedicine.com

\section{A role for private sector laboratories in public health surveillance of antimicrobial resistance}
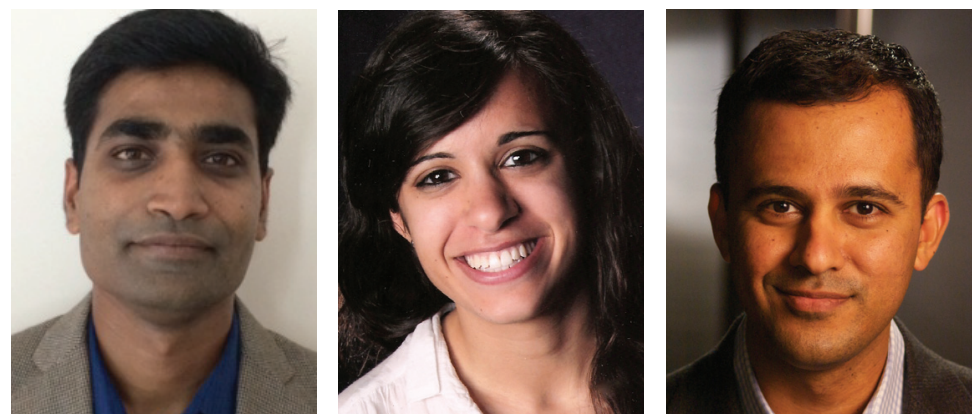

Sumanth Gandra', Anjali T Merchant ${ }^{1}$ \& Ramanan Laxminarayan* ${ }^{* 1,2,3}$

First draft submitted: 7 April 2016; Accepted for publication: 13 April 2016; Published online: 18 May 2016

Policymakers have been slow to respond to the public health threat posed by antimicrobial resistance (AMR) [1], despite increasing health and economic burdens [2]. AMR surveillance data gives shape to an otherwise ill-defined problem, enabling policymakers to more effectively understand, quantify and manage the emergence and spread of resistance. At the patient level, antimicrobial susceptibility testing of clinical isolates can inform prescriber choice of therapeutic treatment [3]. Susceptibility information at the population level allows for the monitoring of resistance trends across space and time, providing evidence on which to base standard treatment practices, antibiotic policies and infection control guidelines [1]. Going beyond susceptibility testing is critical; molecular analysis of global collections of isolates allows for a better understanding of the evolution and spread of resistant strains and genetic elements [4].

Despite recognition of the importance of surveillance data in addressing AMR, the majority of countries lack formal surveillance systems [5]. According to the WHO's Global Antimicrobial Resistance Report, only 22 out of 129 member countries generated national or subnational data between 2000 and 2013 on all seven bacterial pathogens that are commonly associated with community- and hospitalacquired infections, including Escherichia coli, Klebsiella pneumoniae, Staphylococcus aureus, Neisseria gonorrhoeae, Streptococcus pneumoniae, nontyphoidal Salmonella species and Shigella species [5]. Nationallevel data were available mainly in highincome countries in the Americas and the EU, where regional surveillance networks like ReLAVRA (Latin American Antimicrobial Resistance Surveillance Network) and EARS-Net (European Antimicrobial Resistance Surveillance Network) also exist. Few low- and middle-income countries (LMICs), on the other hand, have national AMR surveillance programs. Gaps in the surveillance of antibiotic-resistant bacteria in

\section{KEYWORDS}

- antimicrobial resistance • laboratory capacity • private sector $\bullet$ surveillance

“Policymakers have been slow to respond to the public health threat posed by antimicrobial resistance, despite increasing health and economic burdens." 
“...private laboratory networks and hospitals generate a mine of information that can be tapped in support of antimicrobial resistance surveillance efforts.”
sub-Saharan Africa and South and Southeast Asia are particularly problematic because these regions shoulder disproportionately high burdens of bacterial disease, and consequently a greater potential burden of antibiotic resistance. Given the need to better define the magnitude of resistance in LMICs, in this article we describe how data generated by private sector laboratories can be leveraged in support of public health AMR surveillance.

In LMICs, inadequate laboratory capacity, weak health systems, and financial constraints have hindered the establishment and support of AMR surveillance systems [6]. A survey of 73 public health laboratories conducted by the WHO Regional Office for Africa revealed common challenges to successful laboratory functioning, including lack of trained personnel, old or poorly maintained equipment, erratic supply of water or electricity, a dearth of quality reagents, the absence of or noncompliance with established standard operating procedures (SOPs) and inadequate internal and external quality assurance systems [7]. In Ghana, less than a quarter of the 693 laboratory personnel surveyed from 205 public sector laboratories had received technical training [8]. Preconceptions about the quality of diagnostic laboratory services contribute their underutilization, perpetuating a reliance on less accurate empirical diagnosis at the expense of AMR susceptibility testing [9]. In the absence of a coordinated surveillance strategy, differences in SOPs between laboratories may also affect the comparability of data and hinder their integration into a nationwide network. Together, these factors limit the volume and utility of antimicrobial susceptibility data generated by public sector laboratories for AMR surveillance purposes.

The services offered by private sector facilities create opportunities to collect and harness higher quality antimicrobial susceptibility data. In LMICs, rising incomes have driven a demand for quality healthcare services that is often unmet by public sector infrastructure, given the resource constraints alluded to above [10]. In India and Pakistan, the private sector accounts for more than $70 \%$ of healthcare delivery [11,12], making it a particularly rich source of data. As in the public sector, healthcare facilities in the private sector generate antimicrobial susceptibility data every day in clinical microbiology laboratories for the primary purpose of guiding treatment decisions for individual patients. Accreditation by national and international agencies can serve as a proxy for data quality, indicating that laboratories have undergone quality assurance and proficiency testing. Accredited laboratories in LMICs tend to be predominantly from the private sector. In India, the private sector constitutes $98 \%$ of the 576 medical laboratories accredited by the National Accreditation Board for Testing and Calibration Laboratories (NABL) [13]. Similarly, in South Africa, among the 376 SANAS (South African National Accreditation System)-accredited medical laboratories, more than $80 \%$ come from the private sector [14]. The majority of these accredited private sector laboratories in India and South Africa belong to organized corporate entities and are equipped with better laboratory infrastructure, trained staff, automated methods for species identification and susceptibility testing, and information systems to store and manage data. Data generated by accredited private laboratories may thus represent a means by which to fill the information void left by weak public laboratory systems in LMICs.

The ResistanceMap project [15] demonstrates the utility of private laboratory network data in AMR 'data deserts.' The Center for Disease Dynamics, Economics \& Policy established ResistanceMap to remedy the lack of accessible information on resistance, collating and presenting global resistance data in an online repository. Current ResistanceMap data for India comes exclusively from the private sector, as the country has only recently begun the process of establishing formal national-level surveillance networks under the direction of the National CDC and the Indian Council on Medical Research [16]. Accordingly, estimates of AMR to date have largely been derived from data collected in single-center studies. To gain a better understanding of AMR trends at the national scale, ResistanceMap obtained blood culture data from SRL Diagnostics Limited, one of India's largest private laboratory networks. Approximately 5700 private secondary and tertiary care hospitals, community diagnostic laboratories, and home agencies located in 26 (out of 29) Indian states and two (out of seven) union territories serve as collection centers for SRL. Blood cultures drawn from patients are subsequently transported to the nearest reference laboratory for organism 
isolation, identification and antimicrobial susceptibility testing. Aggregation of SRL data of 18,695 blood isolates (out of 135,268 total blood cultures) collected from 2008 to 2014 presented the opportunity to conduct the first long-term retrospective study of trends in antibiotic resistance on a national scale in India. Analysis of the data indicated both high and increasing resistance to last resort antibiotics in India. For example, carbapenem resistance rates among $K$. pneumoniae and E. coli blood isolates rose from 28.6 to $56.6 \%$ and 9.1 to $11.5 \%$, respectively, from 2008 to 2014 . By comparison, of the 41 other countries that also collected invasive isolate data from 2013 or 2014, only three reported carbapenem resistance rates in E. coli greater than 3\%, including Bulgaria (3.5\%), Turkey (5\%) and Vietnam (9\%) [15]. The SRL data thus reveal worryingly high levels of carbapenem resistance at the national level in India, reaffirming the importance of policies to conserve antibiotic effectiveness.

Antimicrobial susceptibility data generated by the private sector can augment AMR surveillance efforts even in LMICs where formal public sector networks exist. In South Africa, ResistanceMap pooled public and private sector antimicrobial susceptibility data collected by the National Health Laboratory Service and the South African Society for Clinical Microbiology to generate more comprehensive national AMR estimates for the years 2012-2014 [15]. Multisector data can add nuance to our understanding of the emergence and spread and resistance, revealing disparate rates of resistance between the public and private sectors. In LMICs, national drug policies often define which types of antibiotics are procured and prescribed in the public sector $[17,18]$. Differences in the pattern of antibiotic consumption between the public and private sector [19] may, thus, result in differences in resistance rates. The ability to identify and define this variation can improve the precision of strategies that seek to address AMR.

While advocating for a role for private laboratory networks in AMR surveillance in LMICs, we nonetheless recognize the limitations of such an approach. Although data obtained from private laboratory networks like SRL Diagnostics Limited reveal patterns at a national scale, the data may not be nationally representative. Currently efforts are underway to collect and incorporate data from multiple private laboratory networks and hospitals into ResistanceMap to increase confidence in AMR estimates. Rates of resistance also tend to differ according to the source of infection (i.e., community vs hospital acquired) and level of care (i.e., primary, secondary or tertiary) [2], yet private laboratory networks may not always have access to or collect this kind of patient information, limiting the accuracy of AMR estimates calculated from the data collected. Soliciting information from private hospitals, however, with regards to patient location at the time of specimen collection (i.e., outpatient, inpatient wards, or intensive care units) provides one mean by which to differentiate between community- and hospital-acquired infections. Finally, concerns about patient privacy, data ownership and hospital reputation may yield a reluctance to share data. Opportunities for collaboration with private networks and hospitals hinge upon clear delineation of how the data will be stored, used, and publicized [3].

Despite these shortcomings, private laboratory networks and hospitals generate a mine of information that can be tapped in support of AMR surveillance efforts. As LMICs respond to the WHO's call for the implementation of broad-based, national-level surveillance systems in support of the newly established Global Antimicrobial Resistance Surveillance System [20], the availability of resources, health priorities, and the level of political commitment will govern the pace of capacity building and system development. In the meantime, data generated from large private diagnostic laboratory networks and hospitals can fill the lacuna in AMR surveillance in LMICs with minimal resources and know how. Even in countries where nationallevel programs have been established, private diagnostic networks can continue to play a role in surveillance, expanding the evidence base on which AMR policies can be formulated.

\section{Financial \& competing interests disclosure}

$S$ Gandra and $R$ Laxminarayan are supported by ResistanceMap project, which is funded by the Bill \& Melinda Gates Foundation. The authors have no other relevant affliations or financial involvement with any organization or entity with a financial interest in or financial conflict with the subject matter or materials discussed in the manuscript apart from those disclosed.

No writing assistance was utilized in the production of this manuscript. 


\section{References}

1 WHO. WHO global strategy for containment of antimicrobial strategy for containment of antimicrobial resistance (2001).

http://scholar.google.com

2 Grundmann H. Towards a global antibiotic resistance surveillance system: a primer for a roadmap. Ups. J. Med. Sci. 119(2), 87-95 (2014).

3 Shaban R, Cruickshank M, Christiansen K, Antimicrobial Resistance Standing Committee. National surveillance and reporting of antimicrobial resistance and antibiotic usage for human health in Australia. Antimicrobial Resistance Standing Committee, Australian Heath Protection Principal Committee: Canberra. www.griffith.edu.au

4 Wong VK, Baker S, Pickard DJ et al. Phylogeographical analysis of the dominant multidrug-resistant $\mathrm{H} 58$ clade of Salmonella typhi identifies inter- and intracontinental transmission events. Nat. Genet. 47(6), 632-639 (2015).

5 WHO. Antimicrobial resistance: global report on surveillance (2014). http://apps.who.int
6 Ndihokubwayo JB, Kasolo F, Yahaya AA, Mwenda J. Strengthening public health laboratories in the WHO African region: a critical need for disease control. African Heal. Monit. (12), 47-52 (2010).

7 Frean J, Perovic O, Fensham V et al. External quality assessment of national public health laboratories in Africa, 2002-2009. Bull. WHO 90, 191A-199A (2011).

8 Bates I, Bekoe V, Asamoa-Adu A. Improving the accuracy of malaria-related laboratory tests in Ghana. Malar. J. 3(1), 1-5 (2004).

9 Petti CA, Polage CR, Quinn TC, Ronald AR, Sande MA. Laboratory medicine in Africa: a barrier to effective health care. Clin. Infect. Dis. 42(3), 377-382 (2006).

10 Anne M. Health care systems in low- and middle-income countries. $N$. Engl. J. Med. 370 (6), 552-557 (2014).

11 Chakraborty D. The private sector's role in achieving universal health coverage in India (May 2015).

http://geneva-network.com

12 Islam A. Health sector reform in Pakistan: future directions. J. Pakistan Med. Assoc. 4, 174 (2002).
13 National Accreditation Board for Testing and Calibration Laboratories. Directory of accredited medical testing laboratories. www.nabl-india.org

14 South African National Accredition System. Directory of accredited facilities. http://home.sanas.co.za/?page_id $=38$

15 CDDEP. ResistanceMap (2016). http://resistancemap.cddep.org

16 Srivastava RK. National policy for containment of antimicrobial resistance India. www.ncdc.gov.in/ab_policy.pdf

17 Department of Health, South Africa. National drug policy for South Africa (1996). http://apps.who.int

18 Central Drugs Standard Control Organization. National list of essential medicines. http://cdsco.nic.in

19 Kotwani A, Holloway K, Chaudhury RR. Methodology for surveillance of antimicrobials use among out-patient in Delhi. Ind. J. Med. Res. 129(5), 550-560 (2009).

20 WHO. Global antimicrobial resistance surveillance system (2015). http://apps.who.int 